\title{
Enhanced Direct Delta Mush
}

Serguei Kalentchouk

skalentchouk@apple.com

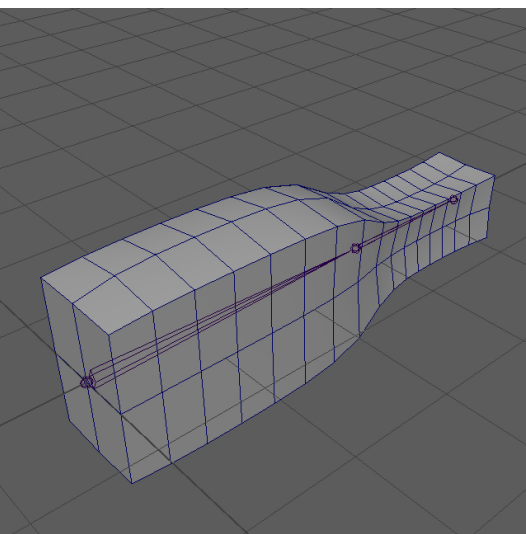

(a) DDM
Michael Hutchinson

michael_hutchinson@apple.com
Deepak Tolani

dtolani@apple.com

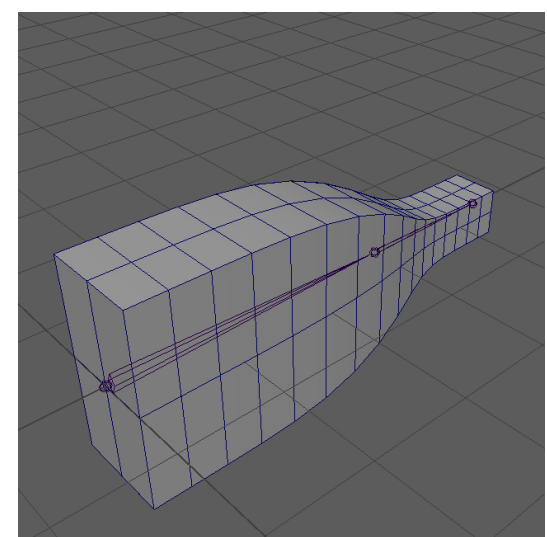

(b) LBS

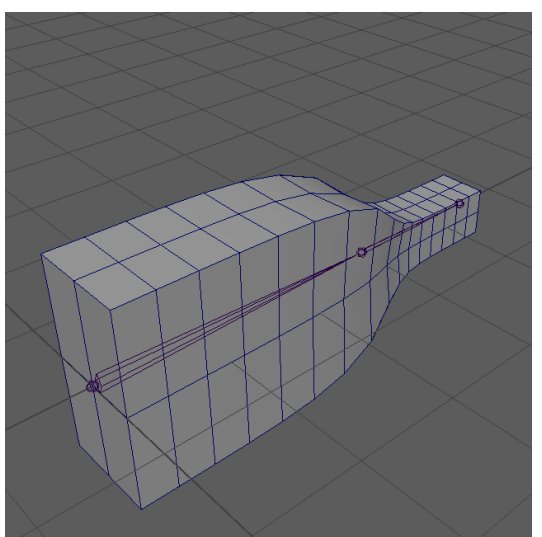

(c) Enhanced DDM

Figure 1: Skinning deformation under non-rigid transformations, with first joint scaled by 2 in the $Y$ axis and the second joint scaled uniformly by 0.5. DDM (a) distorts the original shape and does not capture the full magnitude of the scaling applied to the vertices.

\section{CCS CONCEPTS}

- Computing methodologies $\rightarrow$ Animation.

\section{KEYWORDS}

skinning, deformation, character animation, delta mush

\section{ACM Reference Format:}

Serguei Kalentchouk, Michael Hutchinson, and Deepak Tolani. 2020. Enhanced Direct Delta Mush. In SIGGRAPH Asia 2020 (SA '20 Posters), December 04-13, 2020. ACM, New York, NY, USA, 2 pages. https://doi.org/10.1145/ 3415264.3425464

\section{INTRODUCTION}

Direct Delta Mush is a novel skinning deformation technique introduced by Le and Lewis (2019). It generalizes the iterative Delta Mush algorithm of Mancewicz et al (2014), providing a direct solution with improved efficiency and control. Compared to Linear Blend Skinning, Direct Delta Mush offers better quality of deformations and ease of authoring at comparable performance. However, Direct Delta Mush does not handle non-rigid joint transformations correctly which limits its application for most production environments.

This paper presents an extension to Direct Delta Mush that integrates the non-rigid part of joint transformations into the algorithm.

Permission to make digital or hard copies of part or all of this work for personal or classroom use is granted without fee provided that copies are not made or distributed for profit or commercial advantage and that copies bear this notice and the full citation on the first page. Copyrights for third-party components of this work must be honored

For all other uses, contact the owner/author(s).

SA '20 Posters, December 04-13, 2020, Virtual Event, Republic of Korea

(C) 2020 Copyright held by the owner/author(s).

ACM ISBN 978-1-4503-8113-0/20/11.

https://doi.org/10.1145/3415264.3425464
In addition, the paper also describes practical considerations for computing the orthogonal component of the transformation and stability issues observed during the implementation and testing.

\section{NON-RIGID TRANSFORMATIONS}

"Squash and stretch" is one of the 12 basic principles of animation. In order to accurately model this behavior, it is often necessary to apply non-uniform scale to joint transformations during animation. Unfortunately, Direct Delta Mush (DDM) [Le and Lewis 2019] fails to accurately handle non-rigid joint transformations, as shown in Figure 1a, when compared to similar transformations with Linear Blend Skinning (LBS), shown in Figure 1b. Not surprisingly, the original Delta Mush (DM) [Mancewicz et al. 2014] technique exhibits the same artifacts under non-rigid transformations.

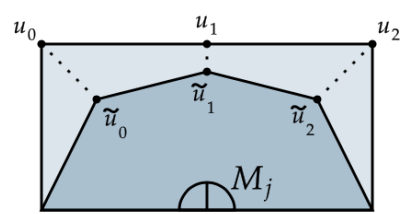

(a) at rest

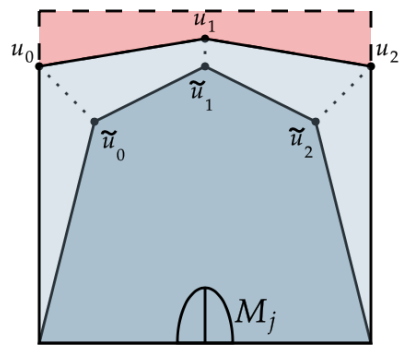

(b) deformed
Figure 2: Delta mush deformation under non-rigid transformation fails to reconstruct the original shape. 


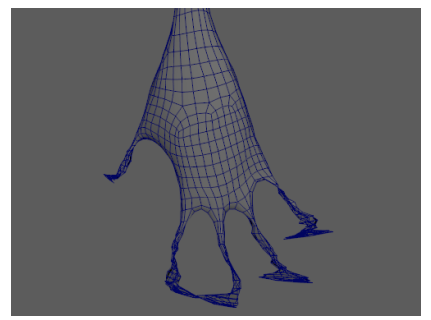

(a)

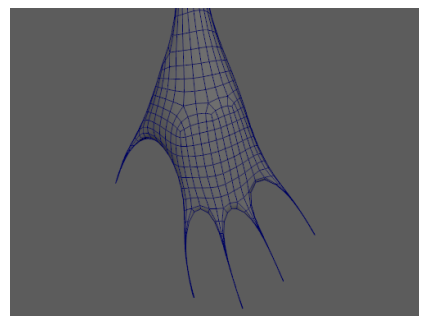

(b)
Figure 3: Smoothed vertices transformed by $\mathrm{R}_{i}$, using a cotangent Laplacian computed with (a) single precision, and (b) with double precision.

Conceptually, DM/DDM applies joint transformations to mesh vertices that have undergone Laplacian smoothing $\tilde{\mathbf{u}}_{i} \in \mathbb{R}^{4}$, and then restores the surface detail lost through smoothing by applying the delta between the smoothed and non-smoothed vertices at rest pose. However, while displacements of smoothed and nonsmoothed vertices under rigid transformation are equivalent, vertex displacements under non-rigid transformation are not, as seen in Figure 2.

The key insight is to compute the displacement of non-smoothed vertices under non-rigid transformation and reintroduce that displacement as a rigid transformation of smoothed vertices.

To that end, the DDM runtime computation is updated as follows. Each joint transformation $\mathbf{M}_{j} \in \mathbb{R}^{4 \times 4}$ is factored into a combined scale and shear matrix $\mathbf{M}_{s j}$ and a rigid only transformation matrix $\mathbf{M}_{r j}$ [Spencer 1991]. Then, for each vertex $\mathbf{u}_{i} \in \mathbb{R}^{4}$ in the rest pose, a displacement under non-rigid transformations is computed as: $\mathbf{d}_{i j}=\mathbf{M}_{s j} \mathbf{u}_{i}-\mathbf{u}_{i}$, where $\mathbf{M}_{s j}$ is applied as a local transformation at each joint. Finally, equation 7 rev. of the DDM algorithm is modified to be:

$$
\left[\begin{array}{cc}
\mathbf{Q}_{i} & \mathbf{q}_{i} \\
\mathbf{p}_{i}^{T} & 1
\end{array}\right]=\sum_{j=1}^{m} \mathbf{M}_{r j} \mathbf{D}_{i j} \Omega_{i j}
$$

where:

$$
\mathbf{D}_{i j}=\left(\begin{array}{cccc}
1 & 0 & 0 & \mathbf{d}_{i j 0} \\
0 & 1 & 0 & \mathbf{d}_{i j 1} \\
0 & 0 & 1 & \mathbf{d}_{i j 2} \\
0 & 0 & 0 & 1
\end{array}\right)
$$

With this formulation, the non-rigid transformation of each influence joint is then weighted using the precomputed weights, producing the desired deformation seen in Figure 1c. This solution supports mixed hierarchies of scale-propagating and scalecompensating joints, which are typical of production rigs in Maya and other digital content creation tools.

\section{NEAREST ORTHOGONAL MATRIX}

To compute the orthogonal component of the vertex transformation, the full model variant of DDM calls for solving the Singular Value Decomposition (SVD) of a $3 \times 3$ matrix $\mathbf{Q}_{i}-\mathbf{q}_{i} \mathbf{p}_{i}^{T}=\mathbf{M}_{i}$, so that $\mathbf{R}_{i}=$ $\mathrm{U}_{i} \mathbf{V}_{i}^{T}$. Performing a full SVD for each vertex comes at a significant computational cost and poses implementation challenges for realtime environments. Instead, we can take advantage of the fact that $\mathbf{M}$ is a square matrix to consider the Polar Decomposition as a solution to the Procrustes problem. Here, $\mathbf{M}_{i}=\mathrm{RS}$, where $\mathrm{R}$ is the nearest orthogonal matrix and $\mathrm{S}$ is a symmetric matrix. To solve for R:

$$
\begin{array}{r}
\mathbf{R}=\mathbf{M}_{i} \mathrm{~S}^{-1} \\
\mathbf{M}_{i}^{T} \mathbf{M}_{i}=\mathbf{S}^{T} \mathbf{R}^{T} \mathbf{R S}=\mathrm{S}^{2} \\
\mathbf{R}_{i}=\mathbf{M}_{i}\left(\mathbf{M}_{i}^{T} \mathbf{M}_{i}\right)^{-1 / 2}
\end{array}
$$

The square root inverse of the symmetric $3 \times 3$ matrix can be efficiently computed using a closed form solution, which can be easily implemented as a compute kernel [Franca 1989]. This formulation provides equivalent results to the full SVD solution at a reduced cost.

\section{PRACTICAL OBSERVATIONS}

While testing with a large range of motion we found instances where the orthogonal matrix $\mathbf{R}_{i}$ contained reflection as well as rotation. This occurs with both the SVD formulation of Le and Lewis, and our square root formulation. These reflections can result in discontinuous motion and undesirable visual artifacts during animation, making it necessary to restrict the solution to rotation matrices. To do so when using SVD, the formula for $\mathbf{R}_{i}$ is modified to be [Higham 1988]:

$$
\mathbf{R}_{i}=\mathbf{U}_{i} \Sigma_{i}^{\prime} \mathbf{V}_{i}^{T}
$$

where:

$$
\Sigma_{i}^{\prime}=\left(\begin{array}{ccc}
1 & 0 & 0 \\
0 & 1 & 0 \\
0 & 0 & \operatorname{det}\left(\mathbf{U}_{i} \mathbf{V}_{i}^{T}\right)
\end{array}\right)
$$

The same correction can be applied to the square root formulation by negating the smallest eigenvalue when the determinant of $\mathbf{M}$ is negative. Note that this requires computing all three eigenvalues using the Stephenson/Sawyers alternative method described by Franca.

Additional testing on more complex geometry revealed that some smoothed vertices were degenerating under the transformation $\mathbf{R}_{i} \mathrm{p}_{i}$, as seen in Figure 3a. This issue was also present in the unmodified implementation of DDM. In order to achieve a stable solution we found it necessary to use double precision arithmetic in the calculation of Laplacian cotangent weights.

\section{REFERENCES}

Leopoldo P. Franca. 1989. An algorithm to compute the square root of a $3 \times 3$ positive definite matrix. Computers \& Mathematics with Applications 18, 5, 459-466. https: //doi.org/10.1016/0898-1221(89)90240-X

Nicolas J. Higham. 1988. Matrix nearness problems and applications. In Proceedings of the IMA Conference on Applications of Matrix Theory. Oxford University Press, $1-27$.

Binh Huy Le and JP Lewis. 2019. Direct Delta Mush Skinning and Variants. ACM Trans. Graph. 38, 4, Article 113 (July 2019), 13 pages. https://doi.org/10.1145/3306346. 3322982

Joe Mancewicz, Matt L. Derksen, Hans Rijpkema, and Cyrus A. Wilson. 2014. Delta Mush: Smoothing Deformations While Preserving Detail. Proceedings of the Fourth Symposium on Digital Production, 7-14. https://doi.org/10.1145/2633374.2633376

Thomas W. Spencer. 1991. Decomposing a Matrix Into Simple Transformations. In Graphics Gems II. Academic Press, 320-323. 